ARTICLE

\title{
Gravity-induced loss of aeration and atelectasis development in the preterm lung: a serial sonographic assessment
}

\author{
Yusuke Hoshino $\mathbb{D}^{1}{ }^{\circledR}$, Junichi Arai ${ }^{1}$, Koji Hirono ${ }^{1}$, Kazushi Maruo ${ }^{2}$, Daigo Kajikawa ${ }^{1}$, Yoshiya Yukitake ${ }^{1}$, Ayako Hinata ${ }^{1}$ and Rena Miura ${ }^{1}$
}

(c) The Author(s), under exclusive licence to Springer Nature America, Inc. 2021

OBJECTIVE: To assess the impact of gravity and time on the changes in the distribution patterns of loss of aeration and atelectasis development in very preterm infants.

STUDY DESIGN: Preterm infants less than 32 weeks gestation were included in this prospective, observational study. Infants were assessed via serial lung ultrasound (LUS) score in four lung zones, performed on days 7, 14, 21, and 28 after birth.

RESULT: Eighty-eight patients were enrolled. There was a significant main effect of gravity $(P<0.001)$ and time $(P=0.01)$ on the LUS score between gravity-dependent lungs and non-dependent lungs. Moreover, there was a significant main effect of gravity $(P=0.003)$ on atelectasis development between the lungs.

CONCLUSION: Gravity and time have an impact on the changes in the distribution patterns of gravity-induced lung injuries in preterm infants.

Journal of Perinatology (2022) 42:231-236; https://doi.org/10.1038/s41372-021-01189-1

\section{INTRODUCTION}

Preterm infants often require prolonged hospitalization with maintaining bed rest. During prolonged bed rest, gravity affects the distribution patterns of fluid in the lungs, which results in nonhomogeneous ventilation, leading to non-uniform distribution patterns of lung injuries, such as dorsal atelectasis or lung edema in the supine position [1, 2]. Thus, these lung injury gradients gradually develop over time. However, serial changes of the distribution patterns have not yet been investigated in very preterm infants.

Clinicians regularly use repeated radiography for the diagnosis of neonatal lung diseases [3, 4]. However, radiography provides an anterior-posterior view, and the use of plain film to assess threedimensional lung injuries is difficult $[5,6]$. Thus, radiography is not suitable for evaluating non-uniform distribution patterns of lung injuries.

Lung ultrasound (LUS) is widely used in critical care settings [7]. LUS is non-invasive, repeatable, and provides real-time information at the bedside. It is also considered a useful modality in the neonatal intensive care unit (NICU) [8-10]. LUS scans can be performed from any direction, which is a major advantage over radiography. Previous studies have demonstrated the utility of LUS for detecting posterior atelectasis development in preterm infants in the supine position $[11,12]$. The ability to perform repeated examinations at the bedside with minimal risk is a major benefit of ultrasound. To date, LUS has been known to be useful for assessing and monitoring lung aeration changes [13].

Understanding the abovementioned mechanism of non-uniform distribution patterns of lung injuries is crucial to avoid the need for excessive oxygen supplementation or prolonged mechanical ventilation, which leads to ventilator-induced lung injuries or the development of bronchopulmonary dysplasia (BPD). We hypothesized that both the gravity effect due to the patients positioning and the time affect the patients spend in that position have an influence on the lung aeration and atelectasis development during the hospital stay. This study aimed to assess the impact of gravity and time on the changes in the distribution patterns of lung injury gradients via serial LUS in very preterm infants. Furthermore, as the LUS score is not yet common in neonatal intensive care settings, we analyzed the reproducibility of image acquisition and inter- and intra-observer image-scoring agreements.

\section{MATERIAL AND METHODS \\ Patients}

This was a single-center, prospective, observational study conducted at a level III NICU at Ibaraki Children's Hospital, Mito, Japan. All preterm infants less than 32 weeks gestation admitted to the NICU from April 2018 to August 2020 were included. Infants with chromosomal abnormalities, major malformations, congenital lung diseases, or congenital heart defects were excluded.

\section{Respiratory management strategies}

During the study period, infants were resuscitated according to Japan's Neonatal Cardiopulmonary Resuscitation guidelines for both term and preterm infants [14], and a single consensus manual for neonatal intensive care was used. Continuous positive airway pressure (CPAP) was the first choice for providing respiratory support if the infants had spontaneous or regular breathing or started to breathe regularly after providing positive pressure ventilation with a bag and mask. Respiratory support was adjusted to maintain a $\mathrm{pH}$ of $7.25-7.35$, transcutaneous oxygen saturation of $88-94 \%$, and $\mathrm{PaCO}_{2}$ of $40-60 \mathrm{mmHg}$. Infants were intubated for persistent apneic episodes, excessively labored breathing, or failure to

\footnotetext{
${ }^{1}$ Department of Neonatology, Ibaraki Children's Hospital, Mito, Japan. ${ }^{2}$ Department of Biostatistics, Faculty of Medicine, University of Tsukuba, Tsukuba, Japan.

email: y-hoshino@md.tsukuba.ac.jp
}

Received: 27 January 2021 Revised: 29 July 2021 Accepted: 10 August 2021

Published online: 20 August 2021 

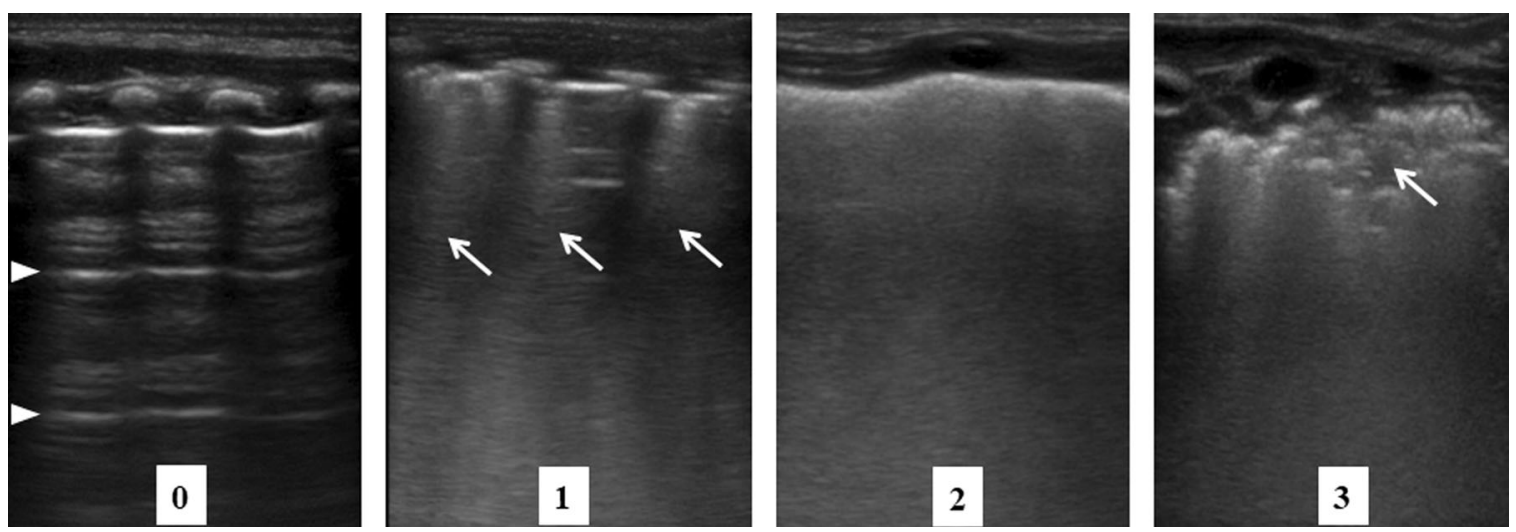

Fig. 1 Lung ultrasound scores. Scores were defined according to the following criteria: 0 , presence of only A-lines (arrowhead); 1 , presence of coalescent or three well-spaced B-lines (arrow) with an A-line; 2, presence of homogeneous and coalescent B-lines with or without consolidations that are limited to the subpleural space; and 3, presence of extended consolidation (arrow).

meet the abovementioned respiratory management policy standards and ventilated using a Babylog VN600 (Dräger, Tokyo, Japan) ventilator. Surfactants were administered based on respiratory difficulty, radiographic findings, the fraction of inspired oxygen to maintain target oxygen saturation values, and stable microbubble rating. In Japan, a non-invasive approach for surfactant administration (e.g., InSurE: intubation-surfactantextubation [15] and LISA: less-invasive-surfactant-administration [16]) is not commonly used to treat extremely preterm infants, especially those between 22 and 24 weeks gestational age, due to the risk of intraventricular hemorrhage from unstable respiratory and circulatory dynamics [17]. Our respiratory management policy is described in Supplemental Text 1.

\section{Positioning}

In our unit, preterm infants with stable hemodynamic status and free from any gastrointestinal diseases are routinely placed in a prone position. Our protocol for preterm infant positioning was as follows: infants without any respiratory difficulty after birth was managed in a supine position and then placed in a prone position within the first $48 \mathrm{~h}$. Infants with respiratory difficulty managed with oxygen supplementation or nasal CPAP were placed in a prone position. Infants under mechanical ventilation were first placed in a supine position and then in a prone position within five days after birth. Once patients were placed in a prone position, the position was maintained until 32 weeks' postmenstrual age.

\section{Lung ultrasound protocol}

We performed serial LUS on days 7, 14, 21, and 28 after birth. LUS was performed using a high-frequency $(6-13 \mathrm{MHz}$ ) linear probe (Sonosite M-turbo, Fujifilm, Japan) on a preset "Superficial" mode. LUS scans were performed by automatically adjusting gain using the dedicated instrument software. The depth was set to $31 \mathrm{~mm}$, and the focus was adjusted according to the patient's size and signs of interest. Each anterior lung was scanned along the midclavicular line, and each posterior lung was scanned along the scapular line to obtain longitudinal scans. To confirm the distribution patterns of lung injury gradients, LUS procedures were performed three hours after the final position changes. With nursery care, LUS was performed in either the supine or prone position. Subsequently, patients were placed in the lateral position, and the opposite lungs were scanned. An LUS score of $0-3$ points was assigned to each area as follows: 0 , presence of A-lines, indicating a well-aerated lung with horizontal multireflection artifacts of the pleura; 1, presence of coalescent or three wellspaced B-lines, indicating lung fluid filling the interstitial and alveolar spaces; 2, presence of homogeneous and coalescent B-lines, indicating severe alveolar edema; and 3, presence of extended consolidation, indicating atelectasis development. Representative images are shown in Fig. 1.

\section{Outcomes}

The main outcome of this study was the impact of gravity and time on the LUS score between gravity-dependent and non-dependent lungs (DL and $\mathrm{NDL}$, respectively). DL was defined as posterior lungs in patients in the supine position or anterior lungs in those in the prone position, and NDL was defined as anterior lungs in patients in the supine position or posterior lungs in those in the prone position. Total LUS scores (0-6 points) were obtained by adding the scores of each lung in the DL and NDL groups, respectively.

To date, LUS scanning protocol for neonates comprising six lung areas (each anterior upper, anterior lower, and lateral lung) [18] with 0-18 points is traditionally applied [19-26]. Thus, we performed additional LUS scanning in our pilot sample of 20 patients, which followed the traditional LUS method. In detail, the DL score was calculated by adding the LUS scores of each posterior upper, posterior lower, and lateral lung in patients in the supine position or anterior upper, anterior lower, and lateral lung in those in the prone position. The NDL score was calculated by adding the scores of each anterior upper, anterior lower, and lateral lung in patients in the supine position or posterior upper, posterior lower, and lateral lung in those in the prone position. Then, total LUS scores (0-18 points) were obtained in the DL and NDL groups, respectively.

\section{Statistical analysis}

To calculate the appropriate sample size, a pilot sample of 20 patients was enrolled on day 7 after birth, which revealed a mean difference of 1.0 in the LUS scores of the DL and NDL groups. The standard deviation (SD) between the two groups was 1.4. Using an a error (two-tailed) of 0.05 and a power of $90 \%$, it was determined that 84 preterm infants were needed to obtain statistically significant results.

Normality was assessed using the Kolmogorov-Smirnov test. Results were expressed as the mean (SD) or median (interquartile range), as appropriate. Independent $t$-test with Bonferroni correction for multiple comparisons was used to compare the LUS scores of the two lung regions (DL and NDL) across each LUS time-point (days 7, 14, 21, and 28) (significance level: $P$-values $<0.05 / 4$ ). Two-way repeated measures of analysis of variance were used to assess the impact of gravity, time, and gravity $\times$ time interaction on the LUS score. A Chi-square test was used to determine the difference in atelectasis between the two lung regions (DL and NDL) across each LUS time-point (day s 7, 14, 21, and 28). A generalized estimating equation (GEE) was used to assess the impact of gravity, time, and gravity $\times$ time interaction on atelectasis (LUS score $=3$ ).

We hypothesized that the impact of gravity on the distribution of lung aeration is related to prematurity. Thus, we also performed a sub-analysis of the LUS score in preterm infants less than 28 weeks gestation.

A neonatal specialist with expert-level experience in LUS (Y.H.) performed all LUS procedures. All LUS images were scored by a neonatal (J.A.) and pediatric specialist (R.M.), and the average scores were used for subsequent analyses. To evaluate reproducibility, Y.H. and H.K. examined 10 additional patients and obtained 40 images independently. Subsequently, another neonatal specialist (A.H.) scored those 80 images. To evaluate interobserver agreement, J.A. and R.M. scored another 80 images independently. To evaluate intra-observer agreement, R.M. re-scored the 80 images three months later. The performers of LUS were blinded from each other's obtained images, and scorers were blinded from each other's measurements. Reproducibility and inter- and intra-observer agreements for scoring single images were evaluated by calculating Cohen's kappa 
$\mathbf{1 0 7}$ infants less than 32 weeks' gestational age

\begin{tabular}{|l|l|l|}
\hline \multicolumn{1}{c|}{} & $\begin{array}{r}\mathbf{1 2} \text { excluded } \\
\mathbf{5} \text { died before } 28 \text { days of life } \\
\mathbf{3} \text { had chromosomal abnormalities } \\
\mathbf{2} \text { had congenital malformations } \\
\mathbf{2} \text { refused of consent }\end{array}$ \\
\cline { 2 - 3 } & $\mathbf{9 5}$ eligible infants & \begin{tabular}{|} 
For $\mathbf{2}$ contraindicated \\
$\mathbf{1}$ had head surgery \\
$\mathbf{1}$ had thoracic drainage
\end{tabular} \\
\cline { 2 - 3 } & $\mathbf{5}$ missed full data collection \\
\hline $\mathbf{8 8}$ included in the study &
\end{tabular}

Fig. 2 Flow chart of study enrollment. According to the exclusion criteria, 88 infants were included, finally.

Table 1. Basic characteristics of the patients.

\begin{tabular}{|c|c|c|c|c|}
\hline Gestational age, weeks, median (IQR) & \multicolumn{2}{|c|}{$\begin{array}{l}\text { Whole population less than } \\
32 \text { weeks }(N=88)\end{array}$} & \multicolumn{2}{|c|}{$\begin{array}{l}\text { Subgroup less than } 28 \text { weeks } \\
(N=38)\end{array}$} \\
\hline Birth weight, g, median (IQR) & 1021 & $(789-1442)$ & 743 & $(650-907)$ \\
\hline Antenatal steroids, $n$ (\%) & 66 & $(75.0)$ & 34 & $(89.5)$ \\
\hline Chorioamnionitis, $n$ (\%) & 31 & $(35.2)$ & 12 & $(31.6)$ \\
\hline Moderate-to-severe BPD, $n(\%)$ & 43 & $(48.9)$ & 33 & $(86.8)$ \\
\hline Postnatal steroids for BPD, $n$ (\%) & 25 & $(28.4)$ & 25 & $(65.8)$ \\
\hline Days of mechanical ventilation, median (IQR) & 2 & $(2-15)$ & 16 & $(4-39)$ \\
\hline Days of hospitalization, median (IQR) & 83 & $(57-103)$ & 109 & $(90-167)$ \\
\hline
\end{tabular}

$B P D$ bronchopulmonary dysplasia, IQR interquartile range.

coefficient with $95 \%$ confidence intervals $(\mathrm{Cls})$. Moreover, inter- and intraobserver agreements for scoring total images ( $0-6$ points) for DL and NDL analysis were evaluated by interclass correlation coefficients with $95 \%$ $\mathrm{Cl}$ and Bland-Altman plots and analysis $[27,28]$.

The sample size was calculated using R statistical software (version 3.6.0; R Foundation for Statistical Computing, Vienna, Austria), and all analyses were performed using SPSS (version 25.0; SPSS Inc. Chicago, IL, USA). $P$-values $<0.05$ were considered statistically significant.

\section{Ethics}

This study was approved by the Institutional Review Board (29IRB-13), and written informed consent was obtained from the parents of each patient.

\section{RESULTS}

During the study period, 107 preterm infants less than 32 weeks gestation were admitted to our unit. In this study cohort, five infants died before reaching 28 days of life. Five infants were excluded based on the exclusion criteria. LUS was contraindicated in two infants (one due to head surgery and one due to thoracic drainage). The parents of two patients did not provide consent for participation in this study, and five patients had incomplete data. Finally, 88 infants were included in the study (Fig. 2). The basic characteristics of the study cohort and characteristics of each LUS time-point are shown in Tables 1 and 2. The classification of BPD was based on the diagnostic criteria published by the National Institute of Child and Human Development at 36 weeks' postmenstrual age [29]. BPD was classified as follows: mild BPD, no oxygen requirement; moderate
$\mathrm{BPD}$, oxygen requirement $<30 \%$; and severe $\mathrm{BPD}$, requiring $\mathrm{CPAP}$ or mechanical ventilation.

On days 7, 14, and 21 after birth, there were modest, but significant differences on the LUS score between the DL and NDL groups (mean [SD] score for each LUS time-point: day 7, 2.6 [1.1] vs. $2.0[1.1], P=0.01$; day $14,2.6[1.4]$ vs. 1.8 [1.2], $P<0.001$; day 21 , 2.5 [1.3] vs. 1.9 [1.3], $P=0.003$, for $\mathrm{DL}$ vs. NDL, respectively). On day 28, no difference between groups was noted (mean [SD]: 2.2 [1.2] vs. 1.8 [1.3], $P=0.064$, for DL vs. NDL). We found a significant main effect of gravity $(P<0.001)$ and time $(P=0.01)$ on the LUS score (Fig. $3 \mathrm{~A})$, but not gravity $\times$ time interaction $(P=0.076)$ (Supplemental Table 1). Atelectasis was significantly occurred in the $\mathrm{DL}$ on days 14 and 21 (percentages of patients between groups: day $7,9.1 \%$ vs. $2.3 \%, P=0.099$; day $14,17 \%$ vs. $2.3 \%, P=$ 0.001 ; day $21,10.2 \%$ vs. $1.1 \%, P=0.018$; day $28,2.3 \%$ vs. $0 \%, P=$ 0.497 for DL vs. NDL, respectively) (Fig. 3B). GEE showed a significant main effect of gravity $(P=0.003)$, but not time $(P=$ $0.257)$ and gravity $\times$ time interaction $(P=0.663)$ on atelectasis (Supplemental Table 2).

In the traditional LUS scanning protocol ( $n=20,0-18$ points), we found a significant main effect of gravity $(P=0.048)$ and time $(P=0.023)$ on the LUS score (Supplemental Fig. 1). There was no significant gravity $\times$ time interaction $(P=0.657)$ (Supplemental Table 1).

In the subgroup analysis of preterm infants less than 28 weeks gestation, there were significant differences on the LUS score between groups on days 7, 14, and 21 (mean [SD] score for each 
Table 2. Characteristics of the patients at each LUS time-point.

\begin{tabular}{|c|c|c|c|c|c|c|c|c|}
\hline \multirow[b]{2}{*}{ Prone position, $n(\%)$} & \multicolumn{2}{|c|}{ Day 7} & \multicolumn{2}{|c|}{ Day 14} & \multicolumn{2}{|c|}{ Day 21} & \multicolumn{2}{|c|}{ Day 28} \\
\hline & 77 & $(88)$ & 74 & $(84)$ & 72 & $(82)$ & 57 & (65) \\
\hline Room air, $n(\%)$ & 12 & (14) & 23 & (26) & 26 & (30) & 34 & (39) \\
\hline Oxygen supplementation, $n(\%)$ & 13 & $(15)$ & 9 & $(10)$ & 14 & (16) & 12 & (14) \\
\hline Nasal CPAP, $n(\%)$ & 38 & $(43)$ & 38 & (43) & 33 & (38) & 31 & (35) \\
\hline Mechanical ventilation, $n$ (\%) & 25 & (28) & 18 & (20) & 15 & (17) & 11 & (13) \\
\hline
\end{tabular}

CPAP continuous positive airway pressure, LUS lung ultrasound.

LUS time-point: day 7, 3.5 [1.2] vs. 2.8 [0.9], $P<0.001$; day 14, 3.8 [1.4] vs. 2.6 [1.0], $P<0.001$; day $21,3.5$ [1.2] vs. 2.8 [1.0], $P=0.011$, for DL vs. NDL, respectively). No differences between groups were observed on day 28 (mean [SD] score: 2.8 [1.1] vs. 2.6 [1.0], $P=$ 0.347 , for DL vs. NDL). No significant gravity $\times$ time interaction was noted $(P=0.004)$ on the LUS score (Supplemental Table 1$)$. The simple main effect of gravity $(P<0.001)$ and time $(P=0.001)$ contributed to this result (Supplemental Fig. $2 A)$. Atelectasis was significantly occurred in the DL on day 21 (percentages of patients between groups: day $7,6 \%$ vs. $1 \%, P=0.108$; day $14,8 \%$ vs. $2 \%$, $P=0.086$; day $21,8 \%$ vs. $1 \%, P=0.028$; day $28,2 \%$ vs. $0 \%, P=$ 0.493 , for DL vs. NDL, respectively) (Supplemental Fig. 2B). GEE showed a significant main effect of gravity $(P=0.009)$, but not time $(P=0.242)$ and gravity $\times$ time interaction $(P=0.497)$ on atelectasis (Supplemental Table 2).

For scoring simple images, weighted kappa coefficient values of 0.84 (95\% Cl: $0.73-0.95), 0.91$ (95\% Cl: $0.89-0.93)$, and 0.84 (95\% Cl: $0.77-0.91)$ indicated good reproducibility and interand intra-observer agreements, respectively. For scoring total images, interclass correlation coefficients values of $0.95(95 \% \mathrm{Cl}$ : $0.9-0.97), 0.95$ (95\% Cl: $0.86-0.96)$, and $95 \%$ limits of agreement of -0.977 to $1.077,-1.343$ to 0.943 also showed the same result for inter- and intra- observer agreements, respectively (Supplemental Fig. 3).

\section{DISCUSSION}

This is the first study in which the impact of gravity and time was assessed on the changes in the distribution patterns of gravityinduced loss of aeration and atelectasis development via serial LUS in very preterm infants. We believe that our findings have the potential for providing appropriate respiratory care, which prevents the development of various complications in preterm lungs.

Most preterm infants are at risk of developing respiratory distress syndrome (RDS) or transient tachypnea of the newborn at birth. In such cases, alveolar collapse or lung edema is homogeneously distributed throughout the lung [19]. During the hospital stay, however, they are placed on bed rest, and lung injury gradients gradually develop over time. We detected the non-uniform distribution patterns of loss of aeration on day 7 after birth, which continued until day 21 . Subsequently, the nonuniform distribution patterns of loss of aeration disappeared from days 21 to 28 . This may be due to the development of respiratory muscle function or the establishment of adequate spontaneous breathing at a later stage. In a previous LUS study for predicting moderate-to-severe BPD [30], the diagnostic accuracy of their LUS scanning protocol, including posterior lung scans, was significantly higher than that of another LUS scanning protocol without posterior lung scans only on day 14 . The result may be due to the impact of lung injury gradients which peaks on day 14 . The incidence of atelectasis may also contribute to the phenomenon; in our study, gravity-induced lung collapse was significantly detected on day 14 or 21 after birth, but not on day 28. This information has the potential to help clinicians for providing appropriate respiratory care in very preterm infants. Moreover,
LUS-guided positioning changes may be effective for reducing the impact of gravity and homogeneous lung aeration, which lead to a decreased need for oxygen supplementation, respiratory support, and BPD development.

Interestingly, we confirmed the main effect of gravity on the LUS score and atelectasis development. However, the main effect of time was only confirmed for the LUS score, but not on atelectasis development. Previous LUS studies also showed the declining trend of LUS scores during the hospital stay [31, 32]. It should be emphasized that the LUS score means the "total" LUS score, which is calculated by adding the regional scores. On the other hand, atelectasis results from a single LUS image $($ score $=3)$. For assessing pulmonary pathologies, the total LUS score or an incidence of atelectasis should be applied, depending on the situation.

In this study, we confirmed the main effect of gravity and time for the distribution patterns of gravity-induced loss of aeration in preterm infants less than 32 weeks gestation. However, we did not find gravity $\times$ time interaction. Conversely, in preterm infants less than 28 weeks gestation, we confirmed the main effect of gravity, time, and gravity $\times$ time interaction. At birth, airway liquid clearance, as a result of sodium ion uptake across the airway epithelium [33], reverses the osmotic gradient and leads to airway liquid reabsorption. However, these mechanisms develop during late gestation and are absent in preterm infants [34]. Thus, premature lungs contain excessive water, which can easily lead to lung edema due to the influence of gravity. The difference in the result between the two gestational groups may reflect the excessive water content. Such lung pathologies were also able to be assessed via serial LUS.

To date, LUS scoring for neonates was traditionally performed at anterior upper, anterior lower, and lateral lungs [18-26], which was first described for the RDS management [18]. The scanning protocol is reasonable because the lung injuries with RDS are homogeneously distributed throughout the lung [19]. However, if we evaluate the distribution patterns of gravity-induced lung injuries, posterior lung scans in patients in supine positions are essential. Thus, we performed the original LUS scanning protocol comprising two anterior and two posterior lungs to evaluate the gravity-induced lung injuries. Our additional LUS scanning protocol analysis based on the traditional protocol (scanning six lung regions with $0-18$ points) was comparable to our original protocol. Our original protocol ensured accurate data analysis. Moreover, it was simple to perform. For the management of preterm infants, the prone position is preferable and frequently applied for improving oxygenation and ventilation, decreasing physiological distress, and improving self-regulation and gastric emptying and residuals [35-38]. Thus, the effect of positions on the distribution patterns of lung injury gradients should be considered in the LUS scanning protocol. At a later stage, a posterior lung scan is considered important for the prediction of developing BPD [30]. Moreover, some LUS studies have emphasized that posterior lung scan provides valuable information and should not be overlooked [31, 32, 39, 40]. To date, a specific LUS protocol or timing has not yet been established for the diagnosis or prediction of various neonatal lung diseases [30]. Thus, an 

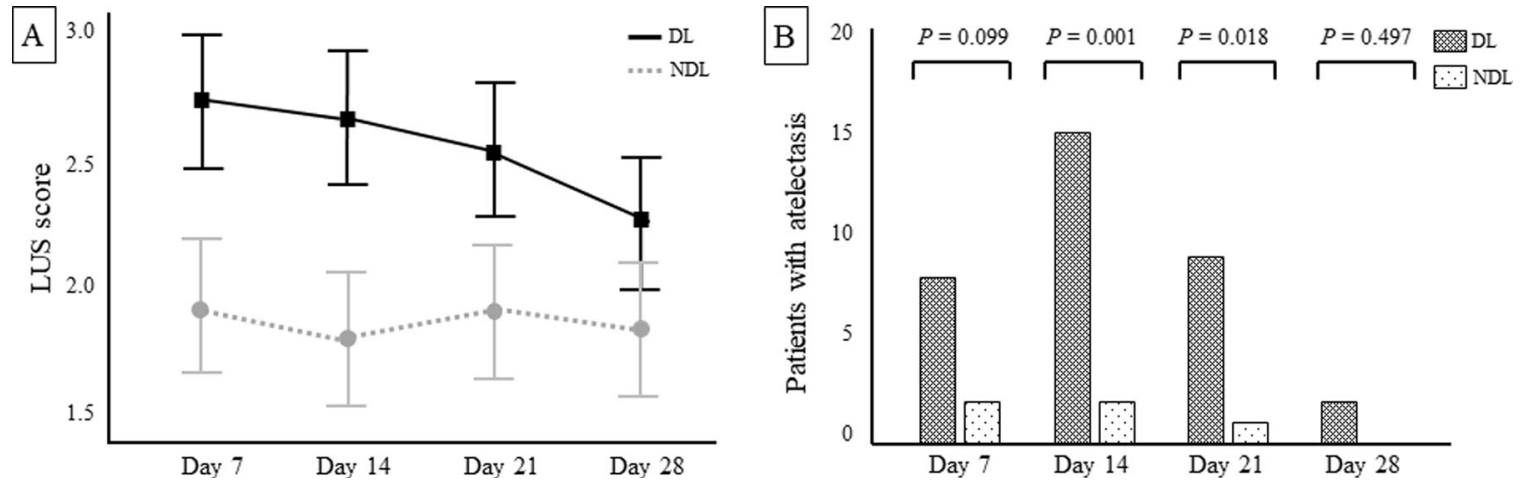

Fig. 3 LUS score and patients with atelectasis $(s c o r e=3)$ at each time-point between the DL and NDL in the whole study cohort. Panel A represents the estimated marginal means of LUS score with $95 \% \mathrm{CI}$. There was a significant main effect of gravity $(P<0.001)$ and time $(P=$ $0.01)$, but not gravity $\times$ time interaction $(P=0.076)$. $P$ values were calculated by two-way repeated measures of analysis of variance. Panel $B$ represents the number of patients with atelectasis. The occurrence of gravity-induced atelectasis was significantly higher in $\mathrm{DL}$ on day $14(P=$ $0.001)$ and day $21(P=0.018)$. $P$ values were calculated by the chi-square test. Cl confidence intervals, DL gravity-dependent lungs, LUS lung ultrasound, NDL non-dependent lungs.

appropriate LUS scanning protocol should be selected based on the projected outcomes.

There are some advantages of LUS compared to other radiological techniques. First, the scoring of LUS images is not complicated. Recently, LUS scoring system has been used for the diagnosis and management of $\operatorname{RDS}[18,20-24]$, predicting the development of BPD $[25,26,30-33,39,40]$, evaluating pulmonary water content [41], and predicting extubation success [42]. In contrast, radiographic evaluation is subjective, which minimizes its generalizability and reproducibility $[43,44]$. Second, LUS can scan from any direction and assess three-dimensional distribution patterns of lung injury gradients. This is a major advantage over radiography because it is difficult to assess such distribution patterns via unidirectional radiation beams. Computed tomography may be useful for the detection of major lung injuries [45]; however, it requires a high degree of radiation exposure. Magnetic resonance imaging is also useful for lung-water gradient evaluation [1, 2], but it cannot be performed at the bedside and requires sedative drugs. Third, LUS is ideal in cases that require repeated measurements without any risk, which is important for the evaluation of changes in lung aeration. Thus, this technique could be used for follow-up examinations. LUS is not difficult to perform and has been recognized as an essential tool for neonatal intensive care by evidence-based guidelines [46]. LUS overcomes several disadvantages of other radiological techniques, enhances our understanding of pulmonary pathology, and can be used as an alternative technique in the NICU.

In this study, we used a high-frequency $(6-13 \mathrm{MHz})$ linear probe that might have limited our findings. However, a previous report on the effect of different probes with micro-linear $(15 \mathrm{MHz})$, phased array sectional $(6-12 \mathrm{MHz})$, and micro convex $(8 \mathrm{MHz})$ probes showed good interrater agreement and reliability [47].

The strength of this study was its confirmation of image acquisition reproducibility and inter- and intra-observer scoring agreements. Inter- $[18,20,21,25,26,36]$ and intra-observer $[25,36]$ LUS image-scoring agreements have previously been evaluated, but reproducibility has not. To date, the LUS scoring system has not been widely adapted for daily neonatal care. In addition, the acquisition of ultrasound images may depend on clinician experience or the technique used [13]. In this study, reproducibility and inter- and intra-observer agreements showed high concordance, indicating that our findings are likely accurate.

This study had some limitations. First, positional changes during nursery care may affect the distribution of lung aeration. A previous study [19] showed that lung lesions were completely re-distributed within $1-2 \mathrm{~h}$ after final position changes. Thus, we performed all LUS procedures three hours after the last position changes to minimize this effect. Second, LUS is based on the interpretation of artifacts generated by the passage of ultrasound beams through lung tissues [48] and cannot detect some lung injuries, such as BPD. Third, clinicians performing LUS were not blinded to the clinical conditions of the patients. Finally, this was a single-center study. Since relatively invasive respiratory management is common in Japan [49], centerspecific treatment strategies might influence the respiratory outcome of preterm infants.

\section{CONCLUSION}

Gravity and time have an impact on the changes in the distribution patterns of gravity-induced lung injuries in preterm lungs. We believe that LUS-guided positioning changes have the potential to improve respiratory outcomes in very preterm infants. Further multicenter studies are needed to provide more evidence and guide the use of LUS in NICUs.

\section{DISCLOSURE}

The abstract had been submitted to the 65th Annual Meeting of the Japan Society for Neonatal Health and Development held online on May 7-9, 2021.

\section{REFERENCES}

1. Adams EW, Counsell SJ, Hajnal JV, Cox PN, Kennea NL, Thornton AS, et al. Magnetic resonance imaging of lung water content and distribution in term and preterm infants. Am J Respir Crit Care Med. 2002;166:397-402.

2. Adams EW, Harrison MC, Counsell SJ, Allsop JM, Kennea NL, Hajnal JV, et al. Increased lung water and tissue damage in bronchopulmonary dysplasia. J Pediatr. 2004;145:503-7.

3. Wilson AC. What does imaging the chest tell us about bronchopulmonary dysplasia? Paediatr Respir Rev. 2010;11:158-61.

4. Walkup LL, Tkach JA, Higano NS, Thomen RP, Fain SB, Merhar SL, et al Quantitative magnetic resonance imaging of bronchopulmonary dysplasia in the neonatal intensive care unit environment. Am J Respir Crit Care Med. 2015; 192:1215-22.

5. Liu J, Chen SW, Liu F, Li QP, Kong XY, Feng ZC. The diagnosis of neonatal pulmonary atelectasis using lung ultrasonography. Chest. 2015;147:1013-9.

6. Liu J, Lovrenski J, Ye Hlaing A, Kurepa D. Neonatal lung diseases: lung ultrasound or chest X-ray. J Matern Fetal Neonatal Med. 2019;20:1-6.

7. Lichtenstein DA. Lung ultrasound in the critically ill. Ann Intensive Care. 2014;4:1.

8. Raimondi F, Yousef N, Migliaro F, Capasso L, De Luca D. Point-of-care lung ultrasound in neonatology: classification into descriptive and functional applications. Pediatr Res. 2018;20:1-8.

9. Fraga MV, Stoller JZ, Lorch SA. Novel technologies for neonatal care: the case of point-of-care lung ultrasonography. Pediatrics. 2018. https://doi.org/10.1542/ peds.2018-1621. 
10. Woods PL. Utility of lung ultrasound scanning in neonatology. Arch Dis Child. 2019;104:909-15.

11. Lovrenski J. Lung ultrasonography of pulmonary complications in preterm infants with respiratory distress syndrome. Ups J Med Sci. 2012;117:10-7.

12. Lovrenski J. Pediatric lung ultrasound-pros and potentials. Pediatr Radiol. 2020;50:306-13.

13. Mojoli F, Bouhemad B, Mongodi S, Lichtenstein D. Lung ultrasound for critically ill patients. Am J Respir Crit Care Med. 2019;15:701-14.

14. Hosono $\mathrm{S}$. The textbook of neonatal cardiopulmonary resuscitation. 3rd ed. https://www.ncpr.jp/eng/course_material.html.

15. Verder H, Albertsen P, Ebbesen F, Greisen G, Robertson B, Bertelsen A, et al. Nasal continuous positive airway pressure and early surfactant therapy for respiratory distress syndrome in newborns of less than 30 weeks' gestation. Pediatrics. 1999. https://doi.org/10.1542/peds.103.2.e24.

16. Göpel W, Kribs A, Ziegler A, Laux R, Hoehn T, Wieg C, et al. Avoidance of mechanical ventilation by surfactant treatment of spontaneously breathing preterm infants (AMV): an open-label, randomised, controlled trial. Lancet. 2011;378:1627-34.

17. Neonatal Research Network of Japan. Neonatal intensive care manual for the infants born at less than 28 weeks of gestation. http://plaza.umin.ac.jp/nrndata/ pdf/NICUManual.pdf.

18. Brat R, Yousef N, Klifa R, Reynaud S, Shankar Aguilera S, De Luca D. Lung ultrasonography score to evaluate oxygenation and surfactant need in neonates treated with continuous positive airway pressure. JAMA Pediatr. 2015. https://doi. org/10.1001/jamapediatrics.2015.1797.

19. Louis $D$, Belen K, Farooqui M, Idiong N, Amer R, Hussain $A$, et al. Prone versus supine position for lung ultrasound in neonates with respiratory distress. Am J Perinatol. 2021;38:176-81.

20. De Martino L, Yousef N, Ben-Ammar R, Raimondi F, Shankar-Aguilera S, De Luca D. Lung ultrasound score predicts surfactant need in extremely preterm neonates. Pediatrics. 2018. https://doi.org/10.1542/peds.2018-0463.

21. Perri A, Riccardi R, lannotta R, Di Molfetta DV, Arena R, Vento G, et al. Lung ultrasonography score versus chest $\mathrm{X}$-ray score to predict surfactant administration in newborns with respiratory distress syndrome. Pediatr Pulmonol. 2018;53:1231-6

22. Perri A, Tana M, Riccardi R, lannotta R, Giordano L, Rubortone SA, et al. Neonatal lung ultrasonography score after surfactant in preterm infants: a prospective observational study. Pediatr Pulmonol. 2020;55:116-21.

23. Gregorio-Hernández R, Arriaga-Redondo M, Pérez-Pérez A, Ramos-Navarro C, Sánchez-Luna M. Lung ultrasound in preterm infants with respiratory distress: experience in a neonatal intensive care unit. Eur J Pediatr. 2020;179:81-9.

24. Vardar G, Karadag N, Karatekin G. The role of lung ultrasound as an early diagnostic tool for need of surfactant therapy in preterm infants with respiratory distress syndrome. Am J Perinatol. 2020. https://doi.org/10.1055/s-0040-1714207.

25. Abdelmawla $M$, Louis $D$, Narvey $M$, Elsayed $Y$. A lung ultrasound severity score predicts chronic lung disease in preterm infants. Am J Perinatol. 2019;36:1357-61.

26. Alonso-Ojembarrena A, Lubián-López SP. Lung ultrasound score as early predictor of bronchopulmonary dysplasia in very low birth weight infants. Pediatr Pulmonol. 2019;54:1404-9.

27. Bland JM, Altman DG. Statistical methods for assessing agreement between two methods of clinical measurement. Lancet. 1986;8:307-10.

28. Bland JM, Altman DG. Measuring agreement in method comparison studies. Stat Methods Med Res. 1999;8:135-60.

29. Jobe AH, Bancalari E. Bronchopulmonary dysplasia. Am J Respir Crit Care Med. 2001:163:1723-9.

30. Alonso-Ojembarrena A, Serna-Guerediaga I, Aldecoa-Bilbao V, Gregorio-Hernández R, Alonso-Quintela P, Concheiro-Guisán A, et al. The predictive value of lung ultrasound scores in developing bronchopulmonary dysplasia: a prospective multicenter diagnostic accuracy study. Chest. 2021. https://doi.org/10.1016/j.chest.2021.02.066.

31. Hoshino Y, Arai J, Miura R, Takeuchi S, Yukitake Y, Kajikawa D, et al. Lung ultrasound for predicting the respiratory outcome in patients with bronchopulmonary dysplasia. Am J Perinatol, 2020. https://doi.org/10.1055/s-0040-1721848.

32. Loi B, Vigo G, Baraldi E, Raimondi F, Carnielli VP, Mosca F, et al. Lung ultrasound to monitor extremely preterm infants and predict BPD: multicenter longitudinal cohort study. Am J Respir Crit Care Med. 2020. https://doi.org/10.1164/rccm.202008-31310C.

33. Jain L, Dudell GG. Respiratory transition in infants delivered by cesarean section. Semin Perinatol. 2006;30:296-304.

34. Hooper SB, Te Pas AB, Kitchen MJ. Respiratory transition in the newborn: a threephase process. Arch Dis Child Fetal Neonatal Ed. 2016;101:F266-F271.

35. Rivas-Fernandez M, Roqué I Figuls $M$, Diez-Izquierdo A, Escribano J, Balaguer A. Infant position in neonates receiving mechanical ventilation. Cochrane Database Syst Rev. 2016. https://doi.org/10.1002/14651858.CD003668.pub4.
36. Vendettuoli V, Veneroni C, Zannin E, Mercadante D, Matassa P, Pedotti A, et al. Positional effects on lung mechanics of ventilated preterm infants with acute and chronic lung disease. Pediatr Pulmonol. 2015;50:798-804.

37. Lubbe W, Van der Walt CS, Klopper HC. Integrative literature review defining evidence-based neurodevelopmental supportive care of the preterm infant. J Perinat Neonatal Nurs. 2012;26:251-9.

38. Chen SS, Tzeng YL, Gau BS, Kuo PC, Chen JY. Effects of prone and supine positioning on gastric residuals in preterm infants: a time series with cross-over study. Int J Nurs Stud. 2013;50:1459-67.

39. Oulego-Erroz I, Alonso-Quintela P, Terroba-Seara S, Jiménez-González A, Rodríguez-Blanco $S$. Early assessment of lung aeration using an ultrasound score as a biomarker of developing bronchopulmonary dysplasia: a prospective observational study. J Perinatol. 2021;41:62-8.

40. Szymański $P$, Kruczek P, Hożejowski R, Wais P. Modified lung ultrasound score predicts ventilation requirements in neonatal respiratory distress syndrome. BMC Pediatr. 2021. https://doi.org/10.1186/s12887-020-02485-z.

41. Zong HF, Guo G, Liu J, Bao LL, Yang CZ. Using lung ultrasound to quantitatively evaluate pulmonary water content. Pediatr Pulmonol. 2020;55:729-39.

42. El Amrousy D, Elgendy $M$, Eltomey $M$, Elmashad AE. Value of lung ultrasonography to predict weaning success in ventilated neonates. Pediatr Pulmonol. 2020;55:2452-6.

43. Moya MP, Bisset GS 3rd, Auten RL Jr, Miller C, Hollingworth C, Frush DP. Reliability of CXR for the diagnosis of bronchopulmonary dysplasia. Pediatr Radiol. 2001;31:339-42.

44. Jobe AH, Steinhorn R. Can we define bronchopulmonary dysplasia? J Pediatr. 2017;188:19-23.

45. Tonson la Tour A, Spadola L, Sayegh Y, Combescure C, Pfister R, Argiroffo CB, et al. Chest $C T$ in bronchopulmonary dysplasia: clinical and radiological correlations. Pediatr Pulmonol. 2013;48:693-8.

46. Kurepa D, Zaghloul N, Watkins L, Liu J. Neonatal lung ultrasound exam guidelines. J Perinatol. 2018;38:11-22.

47. Gomond-Le Goff C, Vivalda L, Foligno S, Loi B, Yousef N, De Luca D. Effect of different probes and expertise on the interpretation reliability of point-of-care Lung Ultrasound. Chest. 2020;157:924-31.

48. Escourrou G, De Luca D. Lung ultrasound decreased radiation exposure in preterm infants in a neonatal intensive care unit. Acta Paediatr. 2016;105:e237-e239.

49. Isayama T. The clinical management and outcomes of extremely preterm infants in Japan: past, present, and future. Transl Pediatr. 2019:8:199-211.

\section{ACKNOWLEDGEMENTS}

We thank Dr. Yoshiro Hitomi, Department of Obstetrics and Gynecology, Mito Saiseikai General Hospital for providing maternal medical information related to pregnancies and births. This work was supported by JSPS KAKENHI (Grant number 20K16839).

\section{AUTHOR CONTRIBUTIONS}

$\mathrm{YH}$ conceptualized and designed this study, contributed to data extraction, conducted the statistical analysis, and drafted the initial manuscript. JA supervised the study design and statistical analysis and contributed to data extraction. $\mathrm{KH}$ contributed to data extraction. KM supervised the statistical analysis. DK and YY supervised the study design. $\mathrm{AH}$ and RM contributed to data extraction. All authors reviewed and revised the manuscript for important intellectual content.

\section{COMPETING INTERESTS}

The authors declare no competing interests.

\section{ADDITIONAL INFORMATION}

Supplementary information The online version contains supplementary material available at https://doi.org/10.1038/s41372-021-01189-1.

Correspondence and requests for materials should be addressed to Y.H.

Reprints and permission information is available at http://www.nature.com/ reprints

Publisher's note Springer Nature remains neutral with regard to jurisdictional claims in published maps and institutional affiliations. 